\title{
Uma ferramenta colaborativa para apoiar a aprendizagem de programação de computadores
}

\section{Leonardo Davi Pereira Machado ${ }^{1}$, Carla Diacui Medeiros Berkenbrock ${ }^{2}$, Guilherme Anselmo Bianeck ${ }^{2}$ and Ivanete Zuchi Siple 3}

\author{
${ }^{1}$ Programa de Pós-Graduação em Computação Aplicada - UDESC and ${ }^{2}$ Departamento de Ciência da Computação - \\ UDESC and ${ }^{3}$ Departamento de Matemática - UDESC \\ *leonardo.davi.machado@gmail.com \\ ${ }^{\dagger}$ carla.berkenbrock@udesc.br \\ ${ }^{\dagger}$ guilhermebianeck@gmail.com \\ ‡ivanete.siple@udesc.br
}

Submetido: 05/10/2017. Revisado: 23/03/2018. Aceito: 15/04/2018.

\begin{abstract}
Resumo
Nos cursos da área da Computação os alunos podem ter dificuldades de aprendizagem nas disciplinas que envolvem programação, o que gera um alto índice de reprovação e desistência nessas disciplinas. Com o intuito de contribuir para a redução dessas dificuldades, este artigo apresenta uma ferramenta colaborativa para apoiar a aprendizagem de programação de computadores por meio de dispositivos móveis. Essa ferramenta adota uma abordagem de aprendizagem colaborativa onde os alunos trabalham usando diferentes estratégias: individualmente seguida por uma atividade em grupo ou diretamente em grupo a fim de trocar experiências para aprender Programção Orientada a Objetos (POO). Este trabalho apresenta um estudo de caso conduzido para demonstrar a aplicabilidade do ferramenta. Os resultados alcançados indicam o potencial da abordagem para apoiar a aprendizagem dos conceitos em programação de computadores.
\end{abstract}

Palavras-Chave: Aprendizagem colaborativa; dispositivos móveis; programação de computador.

\begin{abstract}
In Computer Science programs, students may have learning difficulties in the courses that involve programming, which generates a high rate of failure in these courses. In order to contribute to the reduction of learning difficulties, this paper presents a collaborative tool to support computer programming learning through mobile devices. This tool adopts a collaborative learning approach where students work using different strategies: individually followed by group work, or directly in the group in order to exchange experiences and learn Object Oriented Programming (OOP). This paper also presents a case study to demonstrate the applicability of the tool. The results indicate the potential of the approach to support the learning of computer programming concepts.
\end{abstract}

Key words: Collaborative learning; computer programming; mobile device.

\section{Introdução}

A aprendizagem de programação de computadores não é uma tarefa fácil, exige dos alunos o desenvolvimento do raciocínio lógico, a desenvoltura para a resolução de problemas e a capacidade de abstração de conceitos.
Nos cursos de computação, as disciplinas que envolvem a temática de programação de computadores são reconhecidas como difíceis para os alunos resultando em um alto índice de reprovação e evasão Barcelos et al. (2009); da Silva et al. (2009); Priesnitz Filho et al. (2012); Aureliano et al. (2016). 
Diversos pesquisadores têm empreendido esforços para a criação de ferramentas com o intuito de facilitar a aprendizagem em programação de computadores, em alguns casos aplicando, inclusive, a colaboração no contexto acadêmico com o objetivo de obter melhor desempenho na aprendizagem dos alunos Parsons and Haden (2006); Valdivia et al. (2009); Alvarez et al. (2011); Karavirta et al. (2012); Ihantola et al. (2013); Silveira (2016). Entretanto, acreditamos que ainda exista espaço para uma maior investigação e desenvolvimento de recursos para apoiar a aprendizagem de programação. Sobretudo, dos recursos que tornem os processos flexíveis para acomodar diferentes estratégias para atingir o objetivo de aprendizado, facilitando até mesmo a inclusão de alunos com deficiências Carniel et al. (2017), além de incentivar a colaboração entre os alunos.

As organizações em geral, exigem de seus profissionais uma postura colaborativa a fim de que eles contribuam para o desenvolvimento de competências do grupo. Contudo, aprender a programar de forma colaborativa não é uma tarefa fácil, pois de acordo com Castro et al. (2008) os alunos não estão acostumados a trabalhar em grupo para determinadas finalidades as quais envolvem diferentes processos, estratégias, técnicas e tecnologias.

Buscando suprir a necessidade de apoiar o aprendizado em programação de computadores, a fim de reduzir os índices de reprovação, e incentivar a colaboração entre os alunos para prepará-los para a vida profissional foi desenvolvido o aplicativo CLinClass. $\mathrm{O}$ aplicativo foi desenvolvido para smartphones e tablets, além de poder ser disponibilizado na Web. Usando conceitos de colaboração e sala de aula invertida, o aluno pode realizar as atividades independente de sua localização geográfica Berkenbrock and Hirata (2015). O aplicativo incentiva a autonomia dos alunos e a colaboração para a construção do conhecimento.

Este artigo apresenta o aplicativo CLinClass, bem como um estudo de caso realizado na disciplina Programação Orientada a Objetos do curso de Ciência da Computação em uma universidade pública. $\mathrm{O}$ artigo está organizado da seguinte forma: a Seção 2 apresenta o aplicativo CLinClass; a Seção 3 relata o estudo de caso realizado; e por fim na Seção 4 são apresentadas as considerações finais.

\section{O CLinClass}

o Collaborative Learning in Classroom (CLinClass) é uma aplicação móvel para smartphones e tablets que tem por objetivo facilitar a construção de um ambiente de aprendizagem colaborativa. A aplicação tem o código fonte aberto que pode ser obtido no endereço https://github.com/leonardo-machado/CLinClass. O aplicativo foi projetado para ser utilizado em dispositivos que executem os sistemas operacionais Android, iOS ou Windows Phone. $\mathrm{O}$ aplicativo implementa um conjunto de requisitos funcionais e requisitos para apoiar a colaboração e a aprendizagem, tais requisitos são descritos nos trabalhos Machado et al. (2015); Machado, Berkenbrock and Siple (2016).

A arquitetura do aplicativo, conforme apresenta a Figura 1, foi projetada para permitir que o aplicativo possa ser entregue para os diferentes sistemas operacionais encontrados em smartphones e tablets, mantendo o padrão visual e a interação com o usuário. O Mobile Angular UI ${ }^{1}$ é um framework para o desenvolvimento de aplicações móveis utilizando as tecnologias padrões para desenvolvimento de sistemas web, ou seja, HTML, CSS e JavaScript. O framework disponibiliza componentes com os estilos visuais e padrões de interação semelhantes aos componentes que são encontrados nos diferentes sistemas operacionais utilizados nos smartphones e tablets.

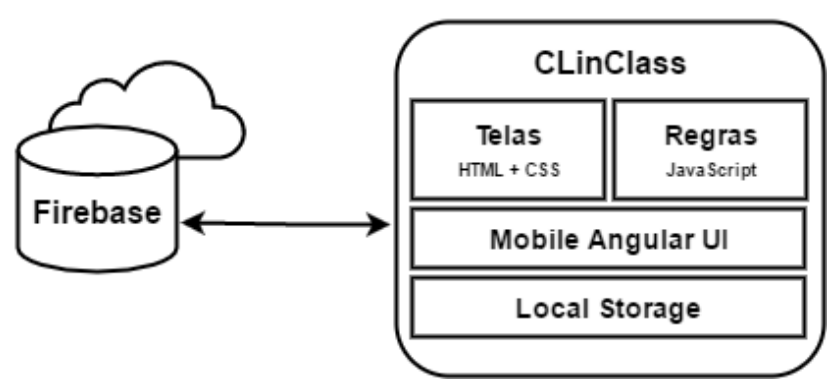

Figura 1: Arquitetura do aplicativo

Dessa forma, o Mobile Angular UI foi utilizado para prover a estrutura básica do aplicativo, facilitando assim o desenvolvimento do CLinClass. As telas foram criadas utilizando a combinação de HTML e CSS, já as regras de negócio e inteligência do aplicativo foram programadas com a linguagem JavaScript.

Como o CLinClass foi desenvolvido com tecnologias web, para realizar o armazenamento de dados dentro do dispositivo foi utilizado o recurso chamado de Local Storage $^{2}$ que permite, no sistema operacional Android, o armazenamento de 2,6 milhões de caracteres (aprox. $2,5 \mathrm{MB}$ ) suficientes para armazenar o texto do enunciado das atividades, as respostas do aluno e as mensagens trocadas. Para o armazenamento geral das informações foi utilizado o serviço chamado Firebase ${ }^{3}$. Este serviço provê um banco de dados na nuvem que armazena as informações em uma estrutura $\mathrm{JSON}^{4}$ e é acessado utilizando uma $\mathrm{API}^{5}$ disponibilizada pelo fornecedor do serviço.

O aplicativo disponibiliza como recurso pedagógico três tipos de atividades que são: questões de múltipla escolha; quebra-cabeça de algoritmo (Parson's Problem); problemas abertos. Os problemas abertos são aqueles em que o aluno tem um campo de texto disponível para colocar livremente sua resposta, dependendo do enunciado do problema a resposta pode ser uma explicação ou o código de um algoritmo. Com as atividades de múltipla escolha o professor pode explorar o conteúdo conceitual da disciplina, permitindo que os alunos exercitem e fixem o conteúdo trabalhado, bem como utilizar esse recurso para fins de avaliação. As Figuras 2 e 3 apresentam um exemplo de atividade do gênero múltipla escolha e de problema aberto.

A atividade do tipo Parson's Problem é uma atividade que se assemelha a um quebra-cabeça cujo o objetivo é reordenar os fragmentos de código a fim de chegar no algoritmo correto Parsons and Haden (2006). As

\footnotetext{
${ }^{1} \mathrm{http://mobileangularui.com/}$

${ }^{2}$ http://www.w3schools.com/html/html5_webstorage.asp

3http://firebase.google.com

4http://www.json.org

5https://pt.wikipedia.org/wiki/Interface_de_programação_de_aplicações
} 


Atividade
Questão 01
Das afirmações abaixo qual é a alternativa
correta com relação ao conceito de
Classe?
o conceito de Classe está presente em
todas as linguagens de programação e é
usado para abrigar variáveis e funções.
O Uma classe é a instância de um objeto,
ela contém atributos e métodos que podem
ser invocados para realizar alguma ação
sobre o objeto.
Classes são estruturas das linguagens
de programação orientadas a objetos para
conter os dados que devem ser
representados e as operações que devem
ser efetuadas com esses dados.
Classe é uma estrutura das linguagens
de programação que possibilita o
armazenamento apenas das variáveis que
um proarama dode acessar.

Figura 2: Múltipla escolha

atividades do tipo Parson's Problem tem por objetivo familiarizar os alunos, principalmente os iniciantes, com a leitura de códigos prontos, estimulando o aluno a encontrar o lugar correto de cada fragmento de código para que o algoritmo chegue ao resultado esperado para aquele programa. As Figuras 4 e 5 apresentam um exemplo desse tipo de atividade com o algoritmo parcialmente montado e identificando um fragmento que não está posicionado corretamente.

Com o CLinClass o professor pode, conforme o conteúdo trabalhado e seu planejamento de aula, adicionar as atividades escolhendo qual o tipo mais apropriado de atividade para cada tema abordado. $\mathrm{O}$ aplicativo permite o professor disponibilizar aos alunos links para os materiais com o conteúdo teórico da disciplina, permitindo que os alunos consultem esse conteúdo ao realizar as atividades, conforme apresenta a Figura 6.

O aplicativo foi projetado para favorecer a colaboração entre os alunos, seguindo a abordagem chamada de Ciclo de Sessão Colaborativa Machado, Berkenbrock, Siple and Hirata (2016). Dessa forma, os alunos trabalham em pequenos grupos e o aplicativo registra as respostas individuais e também uma resposta do grupo para cada uma das atividades desenvolvidas. Assim, na perspectiva do professor o aplicativo permite que sejam observadas as atividades executadas pelo aluno, bem como as respostas individuais e dos grupos, conforme apresenta a Figura 7.

Um elemento importante para a colaboração é a comunicação. Por esse motivo o aplicativo permite, por meio da troca de mensagens de texto, a comunicação entre os alunos e professor, possibilitando que eles esclareçam dúvidas com os pares ou solicitem orientações ao professor. $\mathrm{O}$ aplicativo possibilita também a percepção de trabalho dos outros membros do grupo, sendo possível visualizar quais atividades cada membro já realizou e qual a última vez que as mensagens foram lidas. Nas Figuras 8 e 9 são

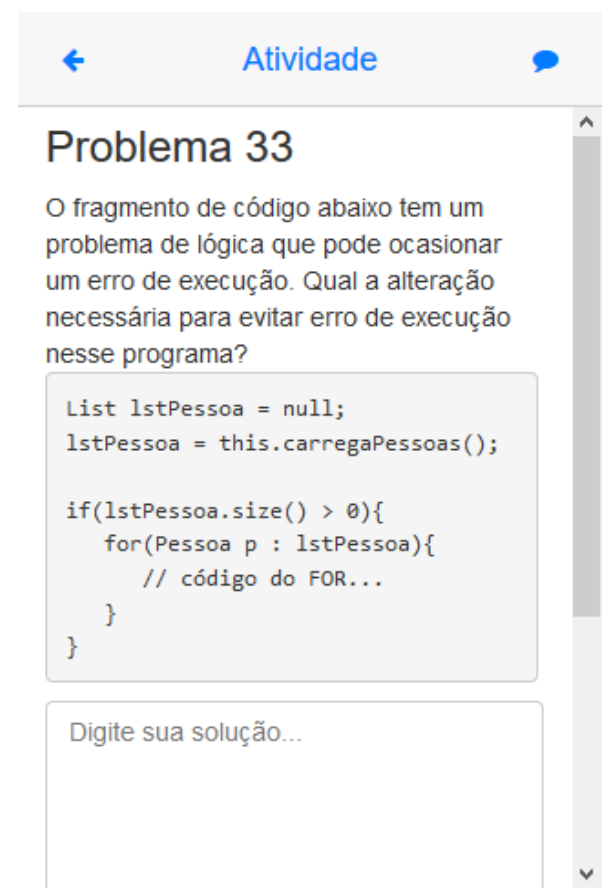

Figura 3: Problema aberto

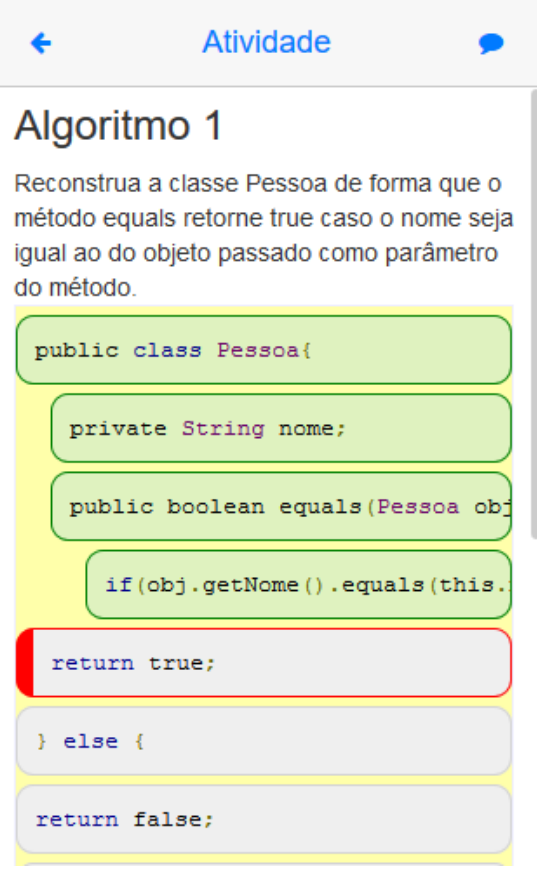

Figura 4: Algoritmo parcialmente montado

apresentadas as telas de mensagens e de informações do grupo.

Após o desenvolvimento do aplicativo CLinClass foi realizado um estudo de caso para avaliar o aplicativo e sua utilização em sala de aula. Na próxima seção é apresentado o estudo de caso conduzido e os resultados obtidos. 


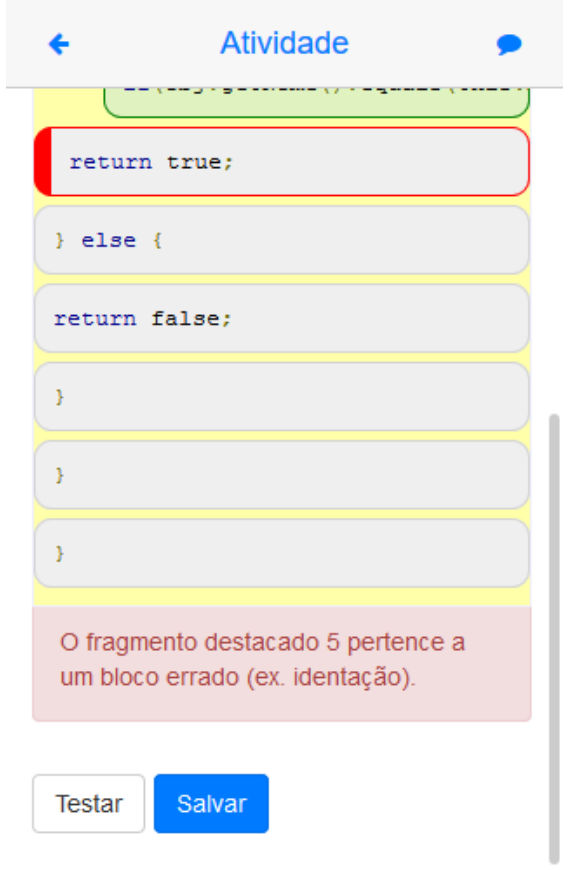

Figura 5: Identificação de erros

\begin{tabular}{ll}
\multicolumn{1}{c}{ Material } & $>$ \\
\hline Aula 1 - Slides & $>$ \\
\hline Aula 2 - Slides & $>$ \\
\hline Validador CPF & $>$ \\
\hline Manual CLinClass & $>$ \\
\hline Aula 3 - Slides & $>$ \\
\hline Aula 4 - Slides & $>$ \\
\hline Aula 5 - Slides & $>$ \\
\hline Aula 6 - Slides & $>$ \\
\hline Aula 7 - Slides & \\
\hline
\end{tabular}

Figura 6: Tela Material

\section{Estudo de Caso}

O estudo foi realizado no primeiro semestre de 2016 com uma turma da disciplina POO0001 - Programação Orientada a Objetos (POO) do curso de Ciência da Computação em uma universidade pública. Esta turma possuía 26 alunos matriculados, sendo que a disciplina de POO foi escolhida pelo alto índice de reprovação verificado na disciplina nos últimos semestres.

Foram realizados quatro encontros com os alunos, o primeiro para fazer um levantamento de informações sobre os alunos e os seguintes para execução dos

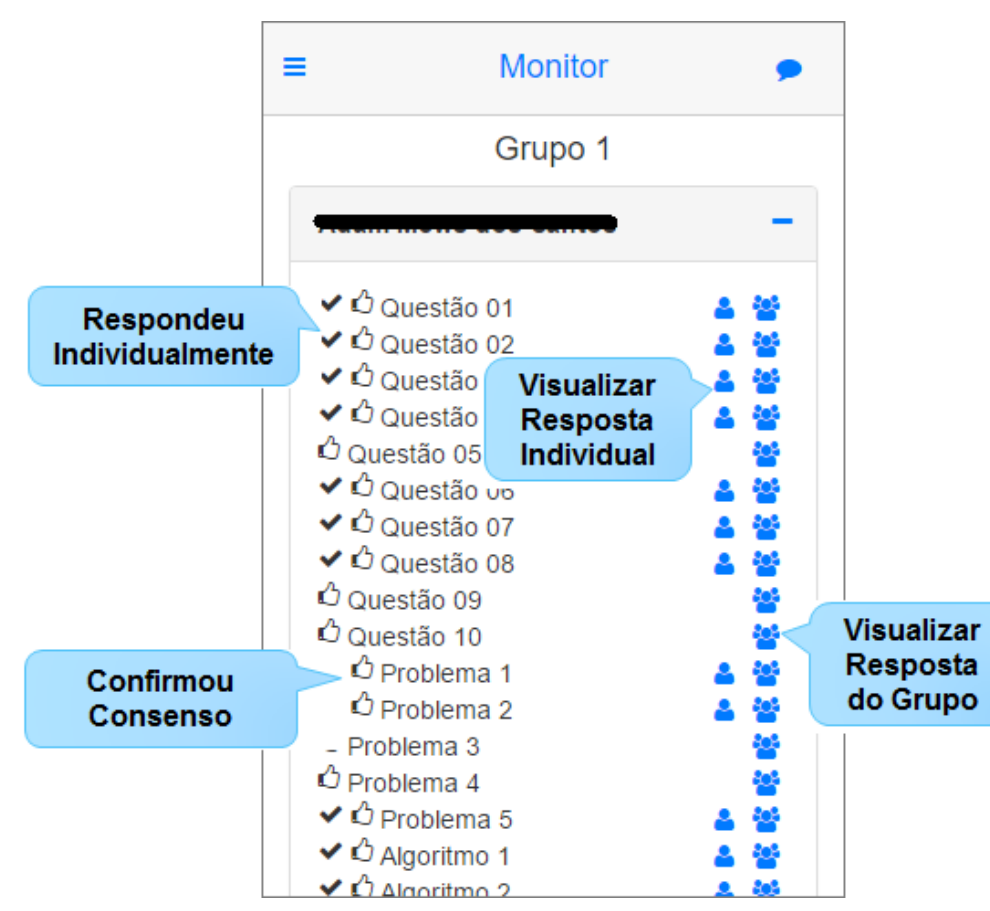

Figura 7: Tela Monitor

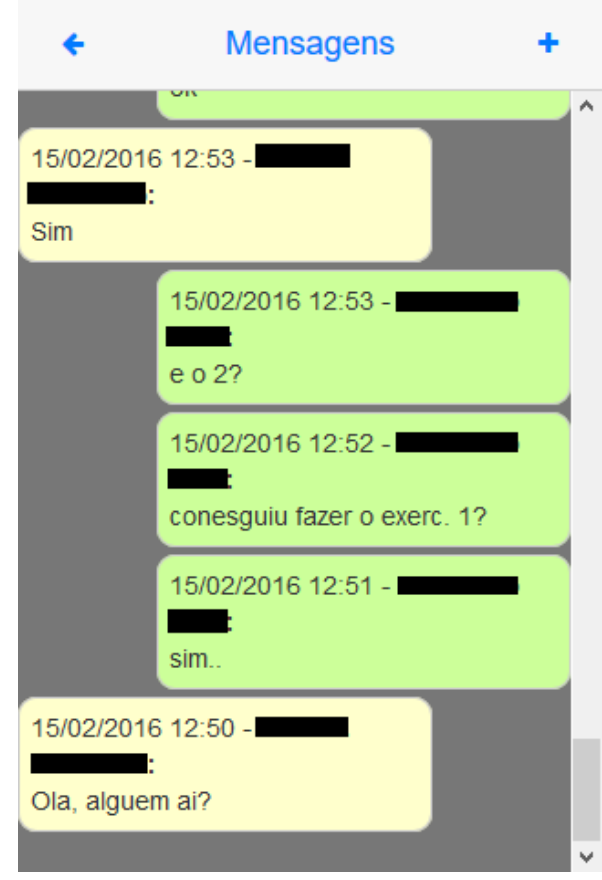

Figura 8: Tela Mensagens

diferentes casos abordados. No primeiro encontro foi explicado aos alunos os objetivos do estudo e como ele seria executado. Nesse encontro aplicou-se um questionário com o intuito de obter o perfil dos alunos quanto ao gênero, idade, aparelhos móveis que possuíam, a configuração desses dispositivos e para quais finalidades eles utilizam os aparelhos móveis, bem como a opinião sobre a utilização dos dispositivos na disciplina de POO.

Todos os integrantes da turma são rapazes, com idade média de dezenove anos. Além disso, todos os 


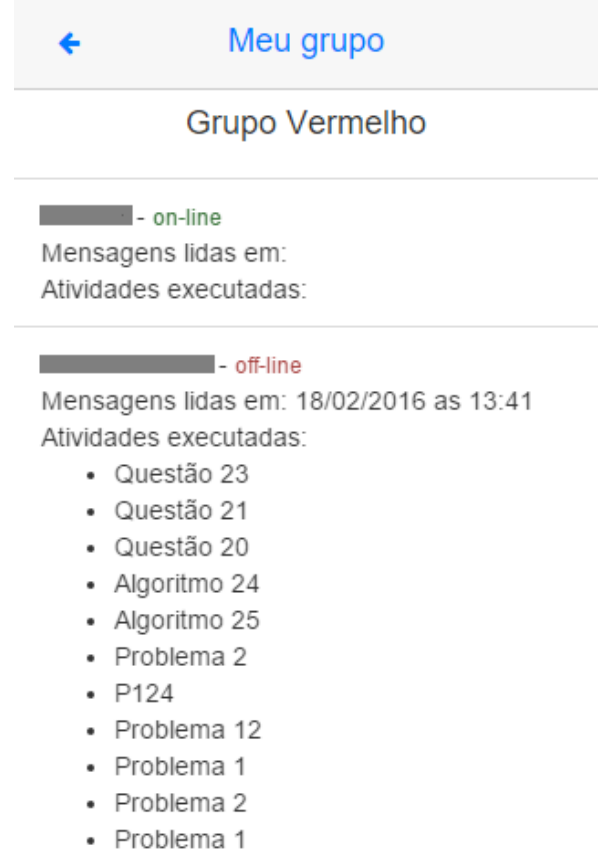

Figura 9: Tela Meu grupo

alunos possuem smartphones e $24 \%$ possuem tablets. Buscou-se também informações sobre a configuração, especialmente o sistema operacional, dos smartphones. Observou-se a predominância do sistema operacional Android em $72 \%$ dos dispositivos, seguido pelo iOS com $16 \%$ e Windows Phone em $12 \%$ dos dispositivos. Para o tamanho da tela observou-se a maior ocorrência dos tamanhos de 4" e 5", 48\% e $36 \%$ respectivamente.

Os alunos foram divididos em cinco grupos de até seis alunos, sendo que os próprios alunos puderam escolher em qual grupo entrar, essa formação de grupos foi mantida nos três subcasos do estudo de caso. No Subcaso 1 os alunos inicialmente fizeram a apresentação de seminários sobre dez tópicos de Orientação a Objetos, depois responderam no aplicativo dez questões de múltipla escolha com o objetivo de revisar os conceitos teóricos apresentados, neste subcaso foi adotada a estratégia na qual os alunos realizariam o trabalho diretamente em grupo.

O Subcaso 2 teve por objetivo trabalhar os conceitos de desenvolvimento de classes e métodos, bem como a resolução de problemas que normalmente ocorrem com programadores iniciantes. Dessa forma, foram passados cinco problemas abertos para os alunos encontrarem uma solução. Inicialmente os alunos trabalharam individualmente extraclasse e depois, em sala de aula, realizaram o trabalho em grupo para chegar a um consenso a respeito da solução para cada problema abordado.

No Subcaso 3 utilizou-se, novamente, a estratégia do trabalho individual seguido de um trabalho em grupo, o objetivo desta atividade foi revisar o conteúdo de estruturas de controle e repetição, sendo utilizados cinco quebra-cabeças de algoritmos (Parson's Problem).

Com a análise das respostas apresentadas pelos alunos e grupos foi possível calcular o índice de acerto individual e dos grupos, conforme apresenta a Tabela 1.

Observa-se nos Subcasos 2 e 3 que, após as discussões em grupo, o índice de acerto do grupo foi melhor que o índice individual dos alunos de cada grupo. No Subcaso 3, por exemplo, os alunos do Grupo 4 (G4) apresentaram individualmente 25 respostas sendo 11 corretas o que representa $44 \%$ de acerto. Contudo, após os debates, o grupo conseguiu acertar as respostas das cinco atividades deste subcaso, representando $100 \%$ de acerto. Tal resultado demonstra que, após os debates em grupo, os alunos conseguem ter uma maior assertividade nas respostas das atividades em relação ao trabalho realizado individualmente.

Após a execução de cada subcaso os alunos responderam um questionário para coletar as suas opiniões quanto as potencialidades e limitações do uso da tecnologia móvel no processo de aprendizagem de programação orientada a objetos e também como o aplicativo contribuiu para a aprendizagem durante o trabalho individual e o trabalho em grupo.

Com a análise das respostas dos alunos, referente as potencialidades do uso da tecnologia móvel no processo de aprendizagem de programação orientada a objetos percebe-se que o uso da tecnologia possibilita a aprendizagem do aluno a qualquer hora e em qualquer lugar, tornando o aprendizado do conteúdo um processo mais leve. O uso do aplicativo CLinClass, segundo os alunos, facilitou o acesso às informações das aulas e possibilitou uma comunicação mais rápida entre os alunos para a realização das atividades propostas. Além disso, o aplicativo possibilitou exercitar a leitura e a escrita de códigos na linguagem de programação Java, que é a linguagem utilizada na disciplina de POO.

Apesar de ter potencialidades, o uso da tecnologia móvel também apresenta algumas limitações. Durante a realização do presente estudo de caso os alunos apontaram algumas dessas limitações. Um exemplo é a falta de sinal de rede $\mathrm{Wi}-\mathrm{Fi}$, em alguns ambientes da universidade, que permita o acesso à internet. Existe a limitação das plataformas móveis que são atendidas, no contexto dessa pesquisa apenas a plataforma Android foi atendida com um aplicativo nativo, para as demais plataformas foi utilizado uma versão web do aplicativo o que demandou a necessidade de conexão com a internet para poder acessá-lo. Os alunos apontam também que o tamanho reduzido da tela do dispositivo limita a interface de interação do usuário. Outra limitação levantada é a ausência de contato direto com o professor e a dificuldade para a escrita de códigos completos.

A respeito de como o uso do aplicativo durante o trabalho individual contribuiu para a aprendizagem, observa-se que o uso do aplicativo contribuiu para trabalhar o conteúdo apresentado pela professora, fazendo o aluno pensar e conversar com os colegas para tirar dúvidas. O uso da ferramenta, segundo os alunos, também incentivou os alunos na busca das respostas, pesquisando o material que foi disponibilizado e cada aluno chegando em suas próprias conclusões.

Durante o trabalho em grupo, segundo os alunos, o uso do aplicativo contribuiu para facilitar a interação entre os participantes do grupo, fornecendo as informações necessárias de cada questão. As alternativas de repostas contidas no aplicativo contribuíram para os debates nos grupos, possibilitando que os alunos obtivessem diferentes pontos de vista sobre o tema abordado em cada atividade. Segundo o relato de um aluno, foi possível chegar a conclusões em grupo de forma mais fácil após ter uma opinião construída durante a etapa de trabalho individual.

Após a conclusão do semestre letivo e fechamento 
Tabela 1: Acertos Individuais X Acertos do Grupo

\begin{tabular}{|c|c|c|c|c|c|c|}
\hline Subcaso & Tipo & G1 & G2 & G3 & G4 & G5 \\
\hline \multirow[t]{4}{*}{1} & Grupo & $90 \%$ & $80 \%$ & $70 \%$ & $80 \%$ & $70 \%$ \\
\hline & & $(9 / 10)$ & $(8 / 10)$ & $(7 / 10)$ & $(8 / 10)$ & $(7 / 10)$ \\
\hline & Individual & $59 \%$ & $42 \%$ & $64 \%$ & $56 \%$ & $37 \%$ \\
\hline & & $(9,5 / 16)$ & $(8 / 19)$ & $(9 / 14)$ & $(9 / 16)$ & $(4,5 / 12)$ \\
\hline \multicolumn{7}{|l|}{2} \\
\hline & Giupo & $(4 / 5)$ & $\begin{array}{l}5070 \\
(2,5 / 5)\end{array}$ & $(4 / 5)$ & $\begin{array}{r}10 \% \\
(3,5 / 5)\end{array}$ & $(3 / 5)$ \\
\hline \multirow{4}{*}{2} & Individual & $96 \%$ & $69 \%$ & $63 \%$ & $44 \%$ & $71 \%$ \\
\hline & & $(22 / 23)$ & (9/13) & $(12 / 19)$ & $(11 / 25)$ & $(10 / 14)$ \\
\hline & & & & & & \\
\hline & Grupo & $100 \%$ & $100 \%$ & $100 \%$ & $100 \%$ & $80 \%$ \\
\hline
\end{tabular}

Fonte: Elaborado pelo autor

das notas foi possível verificar o índice de reprovação ocorrido na turma que foi realizada a intervenção. A Figura 10 apresenta a comparação dos semestres anteriores com o resultado obtido no primeiro semestre de 2016, após a utilização do Ciclo de Sessão Colaborativa e do aplicativo CLinClass.

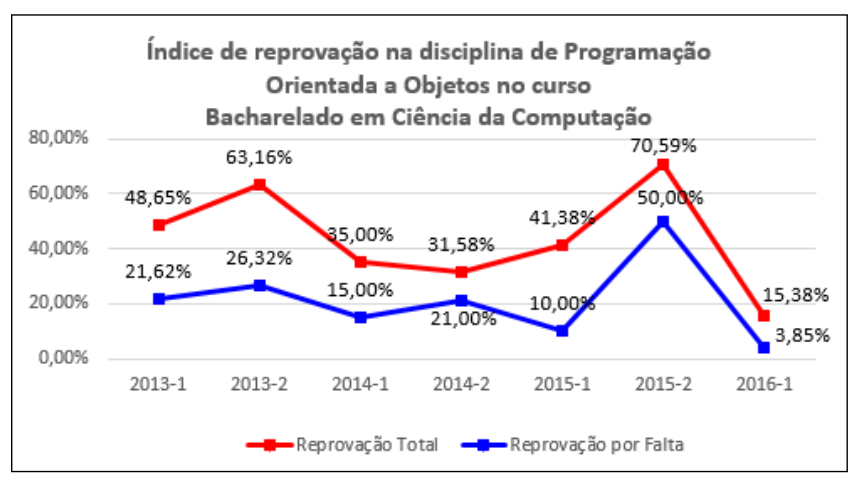

Figura 10: Índice de reprovação após a intervenção

Percebe-se que o índice de reprovação total ocorrido nesse semestre reduziu significativamente, caindo de $70,59 \%$ para $15,38 \%$, é o menor valor registrado dos últimos sete semestres. Sendo, ainda, menos da metade do valor registrado no segundo semestre de 2014 (31,58\%) que era o melhor índice dos últimos três anos. Já o índice de reprovação por falta caiu de $50,00 \%$ para $3,85 \%$, ficando abaixo do menor índice registrado nos últimos três anos. O resultado apresentado é um forte indicativo que a abordagem colaborativa aplicada pode favorecer a aprendizagem dos alunos e consequentemente reduzir o índice de reprovação nas disciplinas relacionadas a programação de computadores. Contudo, ainda não se pode dizer que esse resultado seja exclusivamente pela abordagem aplicada.

\section{Considerações finais}

Este artigo apresentou o aplicativo CLinClass, desenvolvido para apoiar a aprendizagem de Programação Orientada a Objetos em uma abordagem colaborativa, utilizando métodos baseados no Ciclo de Sessão Colaborativo, integrando os alunos e seus docentes em uma ferramenta dinâmica e social.

No contexto desta pesquisa o aplicativo CLinClass foi desenvolvido e utilizado para complementar o processo de aprendizagem colaborativa em turmas de ensino presencial. Contudo, o CLinClass também pode ser utilizado para as formas de ensino a distância e mista, podendo ser adaptado para utilização em outras disciplinas.

O CLinClass amplia as possibilidades do docente, permitindo os alunos trabalharem com problemas abertos, com os quebra-cabeças (Parson's Problems) e com as questões de múltipla escolha. Dessa forma o docente pode, conforme o conteúdo ministrado em aula, escolher quais tipos de atividades utilizar e qual a estratégia a ser aplicada para poder obter um melhor resultado na aprendizagem dos alunos. $\mathrm{O}$ aplicativo inclui o processo de colaboração que envolve a formação de grupos, a comunicação entre os alunos, a percepção do andamento do trabalho dos colegas e a geração do consenso da resposta do grupo.

A ferramenta proporcionou utilização dos dispositivos móveis para o aprendizado da classe e foi considerada como um fator estimulante para os alunos, integrando a tecnologia utilizada diariamente com o ensino.

Com o resultado obtido no estudo de caso percebese que o uso da tecnologia móvel em conjunto com a abordagem colaborativa traz benefícios para o aprendizado dos alunos, estimulando a participação dos alunos nas atividades, a troca de experiências e o trabalho em grupo, resultando em uma redução drástica no índice de reprovação em uma classe e consequentemente atuando na formação do futuro profissional de forma positiva.

Como trabalhos futuros pretende-se aplicar ao aplicativo CLinClass algumas melhorias e novas funções, como por exemplo adicionar um monitoramento sobre as mensagens trocadas pelos alunos permitindo que $o$ professor possa acompanhar o diálogo entre os alunos de cada grupo. Outra melhoria a ser feita é permitir que o professor possa extrair as respostas das atividades individuais e em grupo para realizar as correções e também adicionar um processo de correção automática para as questões de múltipla escolha e para as atividades de quebra-cabeça. 


\section{Referências}

Alvarez, C., Alarcon, R. and Nussbaum, M. (2011). Implementing collaborative learning activities in the classroom supported by one-to-one mobile computing: A design-based process, The Journal of System and Software 84: 1961-1976.

Aureliano, V. C. O., de Azevedo Restelli Tedesco, P. C. and Giraffa, L. M. M. (2016). Desafios e oportunidades aos processos de ensino e de aprendizagem de programação para iniciantes, Workshop sobre Educação em Computação.

Barcelos, R., Tarouco, L. and Bercht, M. (2009). O uso de mobile learning no ensino de algoritmos, RENOTE - Revista Novas Tecnologias na Educação 7(2): 327-337.

Berkenbrock, C. D. M. and Hirata, C. M. (2015). Coerência de cache em sistemas cooperativos com apoio à mobilidade, RBCA - Revista Brasileira de Computação Aplicada 7(1): 17-29. http://dx.doi.org/10.5335/ rbca.2015.3931.

Carniel, A., Berkenbrock, C. D. M. and da Silva Hounsell, M. (2017). Um mapeamento sistemático sobre o uso da comunicação aumentativa alternativa apoiada por recursos tecnológicos, RBCA - Revista Brasileira de Computação Aplicada 9(2): 84-98. http://dx.doi. org/10.5335/rbca.v9i2.6500.

Castro, T., Castro, A. and Fuks, H. (2008). Aprendendo a programar em grupo, SBSC 2008 - Simpósio Brasileiro de Sistemas Colaborativos, pp. 45-54.

da Silva, I., SILVA, I. and SANTOS, M. (2009). Análise de problemas e soluções aplicadas ao ensino de disciplinas introdutórias de programação, IX Jornada de Ensino, Pesquisa e Extensão - JEPEX 2009.

Ihantola, P., Helminen, J. and Karavirta, V. (2013). How to study programming on mobile touch devices: Interactive python code exercises, Proceedings of the 13th Koli Calling International Conference on Computing Education Research, Koli Calling '13, ACM, New York, NY, USA, pp. 51-58. http://doi.acm.org/10.1145/ 2526968. 2526974.

Karavirta, V., Helminen, J. and Ihantola, P. (2012). A mobile learning application for parsons problems with automatic feedback, Proceedings of the 12th Koli Calling International Conference on Computing Education Research, Koli Calling '12, ACM, New York, NY, USA, pp. 11-18. http://doi.acm.org/10.1145/ 2401796.2401798.

Machado, L. D. P., Berkenbrock, C. D. M. and Siple, I. Z. (2016). Desenvolvimento de aplicativos para aprendizagem colaborativa apoiada por dispositivos móveis: uma análise dos requisitos, COMPUTER ON THE BEACH 2016.

Machado, L. D. P., Berkenbrock, C. D. M., Siple, I. Z. and Hirata, C. M. (2015). Utilizando dispositivos móveis para apoiar a aprendizagem colaborativa baseada em problemas, XII Simpósio Brasileiro de Sistemas Colaborativos - SBSC 2015.

Machado, L. D. P., Berkenbrock, C. D. M., Siple, I. Z. and Hirata, C. M. (2016). Uma abordagem colaborativa para aprendizagem de programação orientada a objetos, XIII Simpósio Brasileiro de Sistemas Colaborativos - SBSC 2016.

Parsons, D. and Haden, P. (2006). Parson's programming puzzles: A fun and effective learning tool for first programming courses, Proceedings of the 8th Australasian Conference on Computing Education Volume 52, ACE '06, Australian Computer Society, Inc., Darlinghurst, Australia, Australia, pp. 157-163. http: //dl.acm.org/citation. cfm?id=1151869.1151890.

Priesnitz Filho, W., Abegg, I. and Simonetto, E. (2012). Uma abordagem diferenciada no ensino de algoritmos através da utilização de uma lousa digital, GEINTEC - Gestão, Inovação e Tecnologias 2(2). Disponível em http: //www.revistageintec.net/ portal/index.php/revista/article/view/29.

Silveira, I. F. (2016). A game development-based strategy for teaching software design patterns through challenge-based learning under a flipped classroom approach, Workshop sobre Educação em Computação.

Valdivia, R., Nussbaum, M. and Ochoa, S. (2009). Modeling a collaborative answer negotiation activity using ims-based learning design, IEEE Transactions on Education 52: 375-384. 